\title{
CALIDAD DE LA PREGUNTA DE INVESTIGACIÓN CIENTÍFICA: ANÁLISIS DE LOS ÚLTIMOS 15 AÑOS DE LA REVISTA MEDICINA
}

\author{
QUALITY OF THE SCIENTIFIC RESEARCH QUESTION: ANALYSIS OF THE LAST 15 YEARS OF THE \\ MEDICINE JOURNAL
}

\author{
QUALIDADE DA QUESTÃO DA INVESTIGAÇÃO CIENTÍFICA: ANÁLISE DOS ÚLTIMOS 15 ANOS DA REVISTA
}

MEDICINE

\author{
CARLOS ANDRÉS PEÑAHERRERA OVIEDO \\ Universidad Católica de Santiago de Guayaquil, Guayaquil, Ecuador
}

\begin{abstract}
Introducción: la revista Medicina de la Facultad de Ciencias Médicas de la Universidad Católica de Santiago de Guayaquil (UCSG), recientemente cumplió 20 años de vida institucional; por esta eazón se realizara un análisis de los artículos publicados en la misma, haciendo énfasis en los estudios epidemiológicos y en la correcta elaboración de la pregunta de investigación, base fundamental para un buen estudio, mediante el acrónimo PICOT. Materiales y métodos: se recolectó la información de metadatos de todos los manuscritos publicados en la revista que están disponibles desde su archivo web, y se obtuvo el texto completo de todos los artículos originales de investigación. Resultados: de un total de 543 publicaciones en los últimos 15 años, 232 son artículos de investigación, siendo más frecuentes los de tipo transversal en un 71,6\%. Más del 90\% de los estudios analizados reportan adecuadamente la población, grupo de interés, resultados y tiempo del estudio, mientras que si va el $25,9 \%$ presentan un grupo para comparación, lo que puede ser explicado por la gran cantidad de estudios transversales encontrados. Discusión: los diseños de estudio donde se requirió necesariamente todos los componentes, cumplieron a cabalidad con los requisitos; sin embargo se trata de un pequeño número de artículos. La revista Medicina sigue la tendencia en proporción de artículos originales comparada con otras revistas médicas, y publica en su mayoría estudios con una buena base científica.
\end{abstract}

PALABRAS CLAVE: revistas médicas, pregunta de investigación, estrategia PICOT, investigación científica.

Abstract

Introduction: the Medicine journal recently completed 20 years of institutional life, so we want to provide an analysis of the articles published in this journal, emphasizing the epidemiological studies and the correct elaboration of the research question, the fundamental basis for a good study, by the acronym PICOT. Materials and methods: metadata information was collected from all the manuscripts published in the journal that are available from its web archive, and the full text of all the original research articles was obtained. Results: out of a total of 543 publications in the last 15 years, 232 are research articles, with a $71.6 \%$ being cross-sectional type. More than $90 \%$ of the analyzed studies adequately report the population, interest group, results and time of the study, while only $25.9 \%$ present a group for comparison, which can be explained by the large number of cross-sectional studies found. Discussion: the study designs where all the components are necessarily met complied with the requirements, however it is a small number of articles. The Medicine Journal follows the trend in proportion of original articles compared with other medical journals, and publishes mostly studies with a good scientific basis..

KEYWORDS: medical journals, research question, strategies PICOT, scientific investigation.

Resumo

Introdução: a revista Medicine completou recentemente 20 anos de vida institucional, por isso queremos fornecer uma análise dos artigos publicados nesta revista, enfatizando os estudos epidemiológicos e a correta elaboração da questão de pesquisa, base fundamental para uma bom estudo, pela sigla PICOT. Materiais e métodos: foram coletadas informações de metadados de todos os manuscritos publicados na revista disponíveis em seu arquivo na web, e 0 texto completo de todos os artigos de pesquisa originais foi obtido. Resultados: de um total de 543 publicações nos últimos 15 anos, 232 são artigos de pesquisa, com 71,6\% do tipo transversal. Mais de $90 \%$ dos estudos analisados correctamente relatar população, grupo de interesse, resultados e tempo de estudo, enquanto que apenas $25,9 \%$ tem um grupo para comparação, o que pode ser explicado pelo grande número de estudos transversais encontrado. Discussão: os desenhos de estudo em que todos os componentes são necessariamente cumpridos cumprem os requisitos, no entanto, é um pequeno número de artigos. A revista Medicina segue a tendência na proporção de artigos originais comparados com outros periódicos médicos, e publica principalmente estudos com uma boa base científica..

PALABRAS-CHAVE: médicas revistas, questão de pesquisa, estratégias PICOT, investigação científica. 
INTRODUCCIÓN

La revista Medicina es el órgano difusor oficial de los temas relacionados a la salud por parte de la Universidad Católica de Santiago de Guayaquil, y recientemente cumplió 20 años de vida institucional. ${ }^{1}$ Mantiene un número estable de publicaciones cada año con una salida trimestral en la actualidad. En esta revista se publica todo tipo de artículos médicos, incluyendo estudios epidemiológicos, reportes de caso y revisiones bibliográficas. Es por este motivo que se realizó un análisis retrospectivo de los artículos publicados en la misma, principalmente de los estudios originales de investigación.Todo estudio epidemiológico debe comenzar con una pregunta de investigación; que se encuentre bien construida ya que guiará el resto del proyecto. ${ }^{2}$ Un estudio basado en una buena pregunta de investigación, tendrá una mejor calidad, y sus resultados serán de mayor provecho para el conocimiento científico. ${ }^{3,4}$

La estrategia PICOT responde a un acrónimo que incluye a la población estudiada (P), grupo de intervención o interés (I), grupo de comparación (C), "outcome" o resultado a analizar (O), y el tiempo en el que se desarrolla la investigación (T)..$^{5} \mathrm{El}$ apego a esta estrategia permite elaborar preguntas de investigación de mayor calidad para un estudio determinado. ${ }^{6}$

El objetivo del presente estudio fue, mediante el análisis de todos los artículos originales publicados en la revista Medicina, determinar la cantidad de artículos de investigación publicados cada año, y observar la frecuencia con la que la pregunta de investigación sigue los componentes del PICOT. Análisis similares se han realizado previamente en otras revistas nacionales, ${ }^{7}$ como internacionales, ${ }^{8-10}$ y esta información nos permitirá conocer las características de los estudios de investigación publicados en esta revista, lo que posiblemente refleje el nivel de publicación científica en el Ecuador.

MATERIALES Y MÉTODOS

Se realizó un estudio transversal descriptivo y observacional, en el que se revisaron los archivos en línea de la revista Medicina y se buscó todos los artículos que se publicaron desde el año 2002; en esta base de datos están disponibles los resúmenes y textos completos. Para este estudio se incluyeron los registros en línea desde el volumen 8 , número 2 , hasta el volumen 18 , número 3 ; el cual fue publicado en el 2014. Se excluyeron los artículos de reportes de caso, revisiones bibliográficas y editoriales.

Para obtener las variables se observaron los metadatos de cada número por separado y se anotó el número de publicaciones, divididos en cuatro grupos: editoriales, artículos originales de investigación, reportes decaso, y revisiones bibliográficas o artículos especiales. Para el análisis se revisó cada artículo original individualmente, primero para determinar el diseño de cada estudio, y luego en búsqueda de una pregunta de investigación claramente establecida. Se anotóla presencia o no de cada uno de los 5 componentes de la estrategia PICOT en cada artículo. En este estudio se realizó estadística descriptiva de las variables categóricas. Toda esta información fue recolectada en SPSS Statistics 22 (IBM Corporation) ${ }^{\circledR}$ para el análisis estadístico.

\section{RESULTADOS}

Seencontró un total de 543 manuscritos de todos los tipos entre los años 2002 y 2014, se detalló el tipo de articulo junto al año de publicación en la tabla 1 . Se excluyeron los editoriales, reportes de caso clínico, revisiones bibliográficas y artículos especiales, quedando para ser analizados 232 artículos originales de investigación. La gran mayoría fueron estudios transversales con un total de 166 (71.6\%), seguidos por los ensayos clínicos no randomizados con 27 $(11.6 \%), 26$ estudios de cohorte (11.2\%), 7 ensayos clínicos randomizados (3\%), y 6 estudios de casos y controles $(2.6 \%)$. El total de artículos de cada tipo encontrados en la búsqueda se detalla en la figura 1.

TABLA 1. TOTAL DE MANUSCRITOS PUBLICADOS POR LA REVISTA MEDICINA POR AÑO. PEÑAHERRERA, 2015

\begin{tabular}{lrrrrrr}
\hline AÑO & $\begin{array}{l}\text { EDITORIA- } \\
\text { LES }\end{array}$ & $\begin{array}{l}\text { ARTÍCULOS } \\
\text { ORIGINALE }\end{array}$ & $\begin{array}{l}\text { REPORTES } \\
\text { DE CASO }\end{array}$ & \multicolumn{2}{l}{$\begin{array}{l}\text { REVISIONES } \\
\text { BIBLIOGRÁFICAS }\end{array}$} & TOTAL \\
\hline 2002 & 2 & 18 & 13 & 12 & 45 \\
\hline 2003 & 1 & 13 & 6 & 8 & 28 \\
\hline 2004 & 2 & 13 & 6 & 6 & 27 \\
\hline 2005 & 4 & 20 & 20 & 9 & 53 \\
\hline 2006 & 4 & 23 & 18 & 10 & 55 \\
\hline 2007 & 5 & 23 & 20 & 14 & 62 \\
\hline 2008 & 4 & 26 & 16 & 11 & 57 \\
\hline 2009 & 4 & 21 & 21 & 14 & 60 \\
\hline 2010 & 4 & 24 & 13 & 7 & 48 \\
\hline 2011 & 2 & 15 & 6 & 3 & 26 \\
\hline 2012 & 4 & 25 & 16 & 5 & 50 \\
\hline 2013 & 1 & 3 & 3 & 2 & 9 \\
\hline 2014 & 2 & 8 & 9 & 4 & 23 \\
\hline TOTAL & 39 & 232 & 167 & 105 & 543 \\
\hline
\end{tabular}




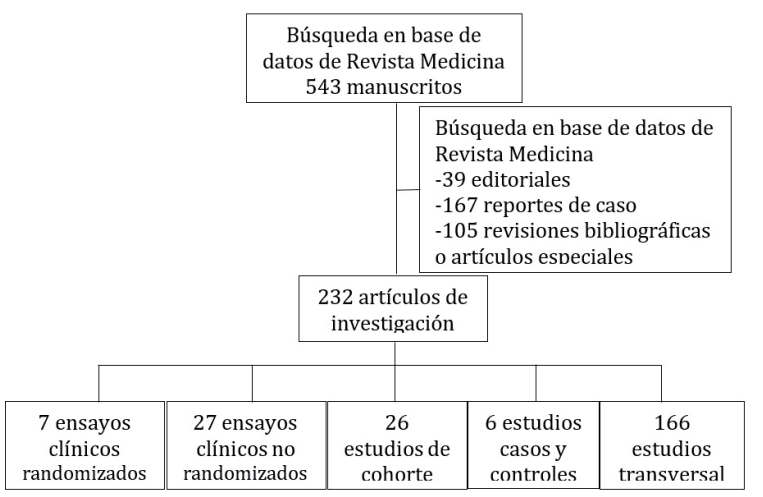

Figura 1. Diagrama de flujo que muestra la inclusión y exclusión de artículos para el análisis, y los tipos de artículos originales encontrados. Peñaherrera, 2015

Se presenta en la figura 2 el total de artículos originales publicados por año en la revista Medicina; mientras que en la figura 3 se muestran los artículos de acuerdo al tipo de estudio, donde se observa claramente el predominio de estudios de tipo transversal. La revista Medicina ha publicado un promedio de 4 números por año de manera ininterrumpida, sin embargo en el año 2013 se encontró menor cantidad de artículos publicados.

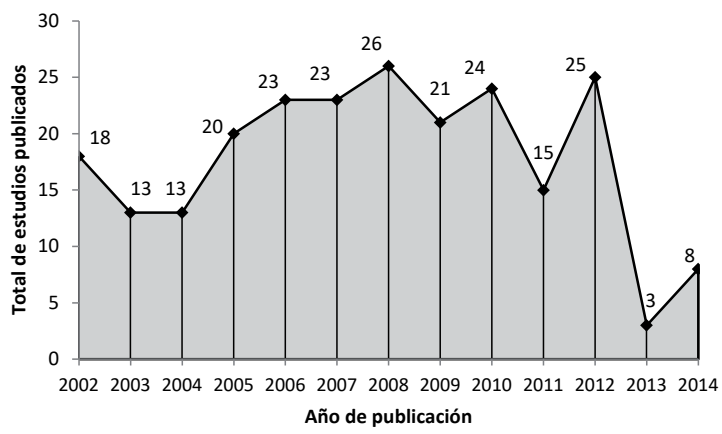

Figura 2. Número de artículos originales publicados en la Revista Medicina, por año. (Nota: en 2013 sólo se publicó un número de la Revista). Peñaherrera, 2015.

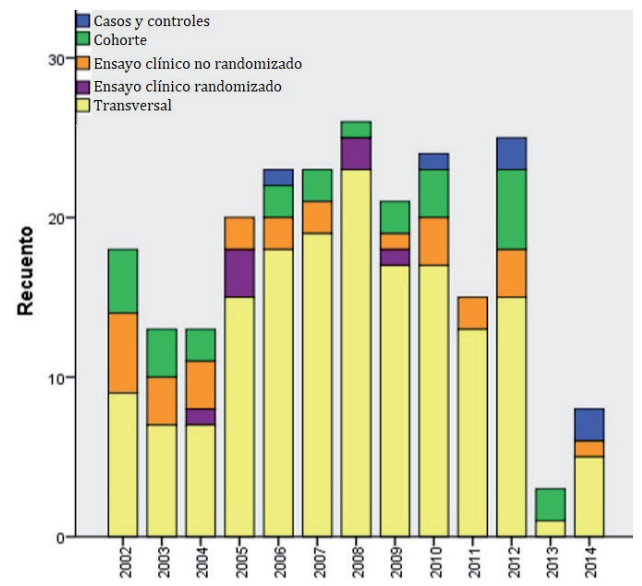

Año de publicación

Figura 3. Artículos originales publicados por año, clasificados por tipo de estudio. Peñaherrera, 2015.
Del cada uno de los 232 estudios analizados, se obtuvo la pregunta de investigación para valorar la presencia de cada componente del acrónimo PICOT en su elaboración. 226 estudios reportaron una población de estudio, mientras que 220 tuvieron bien definido un grupo de intervención o interés. Apenas 60 estudios presentaron un grupo de comparación. El resultado esperado se encontraba expuesto en 162 estudios, y 155 definieron adecuadamente el tiempo de duración de la investigación. Estos resultados, divididos también según el diseño de los estudios, se resumen junto a sus porcentajes en la tabla 2.

TABLA 2. PRESENCIA DE CADA COMPONENTE DE LA ESTRATEGIA PICOT EN LAS PREGUNTAS DE INVESTIGACIÓN DE CADA ARTÍCULO PUBLICADO EN LA REVISTA MEDICINA, CLASIFICADO SEGÚN EL DISEÑO DEL ESTUDIO. PEÑAHERRERA, 2015

\begin{tabular}{lrrrrr} 
& $\mathbf{P}$ & $\mathbf{I}$ & $\mathbf{C}$ & $\mathbf{0}$ & $\mathbf{T}$ \\
\hline Ensayos clínicos & 7 & 7 & 7 & 7 & 7 \\
randomizados & $(100 \%)$ & $(100 \%)$ & $(100 \%)$ & $(100 \%)$ & $(100 \%)$ \\
\hline Ensayos clínicos no & 27 & 27 & 14 & 27 & 26 \\
randomizados & $(100 \%)$ & $(100 \%)$ & $(51,9 \%)$ & $(100 \%)$ & $(96,3 \%)$ \\
\hline Cohorte & 26 & 25 & 12 & 26 & 26 \\
& $(100 \%)$ & $(96,2 \%)$ & $(46,2 \%)$ & $(100 \%)$ & $(100 \%)$ \\
\hline Casos y controles & 6 & 6 & 6 & 6 & 6 \\
& $(100 \%)$ & $(100 \%)$ & $(100 \%)$ & $(100 \%)$ & $(100 \%)$ \\
\hline Transversales & 160 & 155 & 21 & 162 & 155 \\
& $(96,4 \%)$ & $(93,4 \%)$ & $(12,7 \%)$ & $(97,6 \%)$ & $(93,4 \%)$ \\
\hline Todos & 226 & 220 & 60 & 228 & 220 \\
& $(97,4 \%)$ & $(94,8 \%)$ & $(25,9 \%)$ & $(98,3 \%)$ & $(94,8 \%)$ \\
\hline
\end{tabular}

DISCUSIÓN

Los artículos de investigación originales son el tipo más frecuente de manuscritos que se publican en la revista Medicina cada año, con una proporción adecuada si se observa reportes similares de otras revistas biomédicas., ${ }^{7,8}$

En segundo lugar en frecuencia aparecen los reportes de caso clínico, muy comunes en nuestro medio. La gran mayoría de los estudios de investigación son de tipo transversal. Esto puede ser explicado por su relativa facilidad y bajo costo de realización comparado con otros diseños, además de que cierta parte de los artículos originales corresponden a trabajos de graduación, en los que se prefiere un diseño menos complejo.

La importancia de una pregunta de investigación debidamente estructurada es necesaria para la correcta elaboración y desarrollo de un proyecto de investigación. ${ }^{6,11}$ La estrategia PICOT ha demostrado ser una forma eficiente 
de construir la pregunta de investigación, y en el presente trabajo se buscó observar qué tan frecuente se sigue cada uno de sus componentes al momento de formular la pregunta. ${ }^{5,12} \mathrm{La}$ mayoría de artículos reportaron correctamente la población en estudio, el evento de interés, los resultados a observar y el tiempo de ejecución, sin embargo sólo la cuarta parte de los estudios describieron un grupo de comparación. Esto puede ser una consecuencia de la alta frecuencia de estudios transversales obtenida, en los cuales no siempre es necesaria la presencia de un grupo de control. ${ }^{4,13}$ Es importante notar que todos los ensayos clínicos randomizados y los de casos y controles, a pesar de ser pocos, cumplieron a cabalidad con todos los componentes de una buena pregunta de investigación, lo que indica la buena calidad de estos artículos publicados en la revista.

En esta revisión se ha logrado describir las características de los artículos publicados en los últimos 15 años de la revista Medicina, que se encuentran disponibles en el archivo web de la misma.

La revista mantiene tendencias similares en cuanto a publicaciones, comparada con otras revistas de prestigio a nivel nacional e internacional. Debe fomentarse en los investigadores la importancia de establecer correctamente una pregunta de investigación antes de iniciar un proyecto con miras a la publicación, pues esto sin duda mejora la calidad de los artículos y de las revistas donde aparecen.

Finalmente se recomienda realizar análisis similares en otras revistas médicas del país y de la región, para confirmar que se sigan las tendencias de publicación que se han visto reportadas tanto previamente, como en el presente escrito.

\section{REFERENCIAS BIBLIOGRÁFICAS}

1. ConsejoEditorialdela Revista Medicina. Editorial: Dos décadas de la Revista Medicina. Rev Med. 2014;18(2):68.

2. Santos CM da C, Pimenta CA de M, Nobre MRC. The PICO strategy for the research question construction and evidence search. Rev Lat Am Enfermagem. 2007 Jun;15(3):508-11.

3. Martín Rodero H, Galindo Villardón MP. Medicina Basada en la Evidencia y meta-análisis: una aproximación conceptual, histórica y metodológica [Internet] [Máster en Análisis Avanzado de Datos Multivariantes]. [Salamanca]: Universidad de Salamanca; 2011 [cited 2015Jul 8]. Available from: http://gredos. usal.es/jspui/handle/10366/123467

4. Huang X, Lin J, Demner-Fushman D. Evaluation of PICO as a Knowledge Representation for Clinical Questions. AMIA Annu Symp Proc. 2006;2006:359-63.

5. Echevarria IM, WalkerS. To makeyourcase, start with a PICOT question. Nursing (Lond). 2014 Feb;44(2):18-9.

6. Martínez-González A, Sánchez-Mendiola M. La pregunta de investigación en educación médica. Investig En Educ Médica. 2015 Jan;4(13):42-9.

7. Cruz Pareja E, García Santibáñez R, Soria Viteri J. Jerarquía de los diseños epidemiológicos. Revisión de las publicaciones de los 17 años de la. Rev Ecuat Neurol. 2009;18(1-2):111-6.

8. ThabaneL, Thomas T, YeC, PaulJ. Posing theresearch question: not so simple. Can J AnaesthJCan Anesth. 2009Jan;56(1):71-9.

9. Rios LP, Ye C, Thabane L. Association between framing of the research question using the PICOT format and reporting quality of randomized controlled trials. BMC Med Res Methodol. 2010 Feb 5;10(1):11.

10. Borg Debono V, Zhang S, Ye C, Paul J, Arya A, Hurlburt L, et al. A look at the potential association between PICOT framing of a research question and the quality of reporting of analgesia RCTs. BMC Anesthesiol. 2013;13(1):44.

11. Richardson WS, Wilson MC, Nishikawa J, Hayward RS. The well-built clinical question: a key to evidence-based decisions. ACP J Club. 1995 Dec;123(3):A12-3.

12. Riva JJ, Malik KMP, Burnie SJ, Endicott AR, Busse JW. What is your research question? An introduction to the PICOT format for clinicians. J Can Chiropr Assoc. 2012 Sep;56(3):167-71.

13. Fernández SP. Elementos básicos en el diseño de un estudio. Cad Aten Primaria. 1996;3(2):83-5. 